\title{
C. Englisch-Fritz
}

\author{
Spiroergometry in Patients with Pulmonary Hypertension by Lung Fibrosis, \\ Primary Pulmonary Hypertension, Chronic Thrombolic Pulmonary Hypertension \\ and Cystic Fibrosis
}

\begin{abstract}
Die Spiroergometrie ist eine nichtinvasive Messmethode, die ein Verfahren bezeichnet, bei dem unter ergometrischer Belastung die Ventilation und der Gasaustausch gemessen wird. Mit dieser Untersuchungsmethode können die allgemeine körperliche Leistungsfähigkeit und die Zusammenhänge zwischen der Belastung und der Herzförderleistung, dem Sauerstoffverbrauch und der Ventilation der Lunge aufgezeigt werden.
\end{abstract}

Wir haben 11 Patienten mit einer pulmonalen Hypertonie infolge einer Lungenfibrose (LF) mit 11 „gematchten“ gesunden Kontrollpersonen, 39 Patienten mit einer zystischen Fibrose (CF) mit 13 „gematchten“ gesunden Kontrollpersonen und 21 Patienten mit einer primären pulmonalen Hypertonie (PPH) mit $16 \mathrm{~Pa}-$ tienten mit einer chronisch thromboembolischen pulmonalen Hypertonie (CTEPH) mittels Spiroergometrie und Blutgasanalyse untersucht und miteinander verglichen. So konnten wir die leistungslimitierenden Faktoren kardialer, ventilatorischer, metabolischer oder kardiorespiratorischer Art bei den untersuchten Gruppen vergleichen. Durch Korrelationsanalysen mit Lungenfunktions- bzw. Hämodynamikparametern wurde geprüft, welche Ruheparameter einen Vorhersagewert für die maximale Sauerstoffaufnahme (peak $\mathrm{VO}_{2}$ ) haben. Darüber hinaus wurden die komplexen Gasaustauschstörungen der Erkrankungen beschrieben und es wurden die Unterschiede zwischen Patienten mit primärer pulmonaler Hypertonie ( $\mathrm{PPH}$ ) und Patienten mit chronisch thromboembolischer pulmonaler Hypertonie (CTEPH) untersucht.

Bei LF-Patienten war die peak $\mathrm{VO}_{2}$ auf $46 \%$ der Norm signifikant gegenüber den Kontrollpersonen reduziert. Die Leistungslimitierung beruhte überwiegend auf ventilatorischen Faktoren, wei-

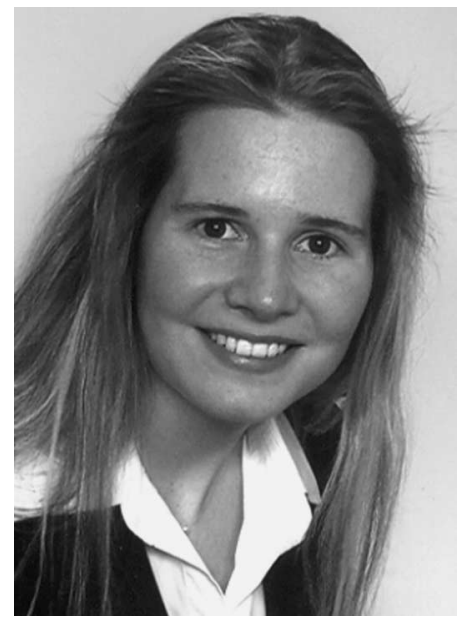

Dr. med. Christina EnglischFritz

terhin aber auch auf zirkulatorische Faktoren, eingeschränkte Atmungseffektivität, erniedrigte Diffusionskapazität und Ventilations-Perfusions-Verteilungsstörungen. Diese schweren Störungen im Gasaustausch führen zu enormer Totraumventilation einerseits und Hypoxämie andererseits, die beide zu der stark eingeschränkten peak $\mathrm{VO}_{2}$ beitragen.

Bei unseren CF-Patienten war die peak $\mathrm{VO}_{2}$ auf $73 \%$ der Norm signifikant gegenüber den Kontrollpersonen reduziert. Die Leistungslimitierung beruhte auf eingeschränkte Lungenfunktionsparameter (Einsekundenausatmungskapazität, Vitalkapazität und Resistance), erniedrigte Körpergröße und Körpergewicht und erniedrigter Diffusionskapazität.

\begin{abstract}
Institutsangaben
Pneumologische Klinik Waldhof/Elgershausen

Anmerkung

Beste klinische Arbeit

Korrespondenzadresse

Dr. med. Christina Englisch-Fritz · Pneumologische Klinik Waldhof/Elgershausen · 35753 Greifenstein
\end{abstract}


Weiterhin zeigten die Patienten eine erhöhte Totraumventilation und ein erhöhtes Atemäquivalent für $\mathrm{CO}_{2}$. CF-Patienten weisen also erhebliche Gasaustauschstörungen auf, die zu gesteigerter Totraumventilation und zu Hypoxämie führen.

Bei den PPH-Patienten war die peak $\mathrm{VO}_{2}$ auf $55 \%$ der Norm und bei den CTEPH-Patienten auf $43 \%$ der Norm reduziert. Die Patienten wiesen eine zirkulatorische Leistungslimitierung auf. Bei unseren PPH-Patienten korrelierten der mittlere pulmonalarterielle Druck, der zentralvenöse Druck, die zentralvenöse Sauerstoffsättigung, die arterielle Sauerstoffsättigung, und der Cardiac Index als hämodynamische Ruheparameter signifikant mit der peak $\mathrm{VO}_{2}$. Bei den CTEPH-Patienten korrelierte dagegen keiner der hämodynamischen Ruheparameter signifikant mit der peak $\mathrm{VO}_{2}$. CTEPH-Patienten wiesen ein signifikant höheres $\mathrm{EQCO}_{2}$ auf als PPH-Patienten. Bei den PPH-Patienten korrelierte dieser Parameter am besten mit der peak $\mathrm{VO}_{2}$. Dahingegen korrelierte dieser bei den CTEPH-Patienten nicht mit der peak $\mathrm{VO}_{2}$.

Als bester Parameter zur Unterscheidung zwischen PPH- und CTEPH-Patienten erwies sich die alveolo-arterielle $\mathrm{CO}_{2}$-Differenz $\left(\mathrm{AaDCO}_{2}\right)$.
PPH-Patienten zeigten eine $\mathrm{AaDCO}_{2}$ von ca. $0,57 \%$ und CTEPHPatienten von $1,15 \%$ in Ruhe. Wenn eine $\mathrm{AaDCO}_{2}$ von $>1 \%$ als Marker einer CTEPH zugrunde gelegt wird, so erlaubt dieser die Abgrenzung von einer PPH mit einer Spezifität von $95 \%$ und einer Sensitivität von $75 \%$.

Bei maximaler Belastung betrug die $\mathrm{AaDCO}_{2}$ bei den PPH-Patienten $0,52 \%$ und bei den CTEPH-Patienten $1,31 \%$. Wenn nun eine $\mathrm{AaDCO}_{2}$ ebenfalls von $>1 \%$ als Marker einer CTEPH zugrunde gelegt wird, so erlaubt dieser die Abgrenzung von einer PPH mit einer Spezifität von $90 \%$ und einer Sensitivität von $88 \%$.

So lassen sich PPH-Patienten und CTEPH-Patienten in Ruhe und unter maximaler Belastung mit relativ hoher Sensitivität und Spezifität voneinander unterscheiden.

Insgesamt erwies sich die Spiroergometrie bei Patienten mit schweren bronchialen, parenchymatösen und vaskulären Lungenkrankheiten als sichere und zuverlässige Methode zur Erfassung der maximalen Sauerstoffaufnahme und der leistungslimitierenden Faktoren. 